$\mathrm{DTP} / 99 / 57$, gr-qc/0002063

\title{
The Dynamics of Curved Gravitating Walls
}

\author{
Filipe Bonjour ${ }^{1},{ }^{*}$ Christos Charmousis $^{2},^{\dagger}$ and Ruth Gregory ${ }^{2 \ddagger}$ \\ ${ }^{1}$ Departamento de Matemética Aplicada, IMECC, Universidade Estadual de Campinas, \\ CP 6065, 13081-970 Campinas SP, Brazil. \\ 2 Centre for Particle Theory, Durham University, South Road, Durham, DH1 3LE, U.K.
}

(November 21, 2018)

\begin{abstract}
We examine the dynamics of a self-gravitating domain wall using the $\lambda \Phi^{4}$ model as a specific example. We find that the Nambu motion of the wall is quite generic and dominates the wall motion even in the presence of gravity. We calculate the corrections to this leading order motion, and estimate the effect of the inclusion of gravity on the dynamics of the wall. We then treat the case of a spherical gravitating thick wall as a particular example, solving the field equations and calculating the corrections to the Nambu motion analytically for this specific case. We find that the presence of gravity retards collapse in this case.
\end{abstract}

PACS numbers: 98.80.Cq, 11.27.+d, 04.40.-b

gr-qc/0002063

Typeset using REVTEX

\footnotetext{
*E-mail address: Bonjour@ime.unicamp.br

$\dagger$ E-mail address: Christos. Charmousis@durham.ac.uk

$\ddagger$ E-mail address: R.A.W.Gregory@durham.ac.uk
} 


\section{INTRODUCTION}

Over the past few decades, topological defects have become a familiar class of objects in many areas of physics. In cosmology, defects are believed to arise generically during phase transitions in the early Universe, and have been notably invoked to account for the anisotropies which seeded cosmological structures [1]. Despite the discouraging discovery that the power spectrum of global strings does not agree with observations of the COBE satellite [2], recent claims of a nongaussian component in the microwave background [3] (see also [任), and the improved agreement of the spectrum predictions for models of cosmic strings with a cosmological constant [5] (whose best fit coincides with the value of $\Lambda$ determined from type Ia supernovae [6]) suggest that it may be still too early to discard them as the source of cosmic structure.

Domain walls are defects that arise when the phase transition occurs by the breakdown of a discrete symmetry. They correspond to solitons in $1+1$ dimensions which are extended in two spatial dimensions to form a wall structure. Because static wall solutions depend only on one coordinate (the distance from the wall's core), they can often be found analytically in the absence of gravity, and perturbatively analytically in its presence. In a cosmological context, it was soon realised [0] that the existence of domain walls with $\eta \gtrsim 1 \mathrm{MeV}$ must be ruled out, because a network of such defects would rapidly evolve to dominate the energy of the Universe. Nevertheless, domain walls remain intrinsically interesting objects to study, for instance for their properties as hypersurfaces; in cosmology, domain walls have been proposed as a realization of our universe in higher dimensions [8], and are currently being explored as a possible resolution of the hierarchy problem [9].

When considering 'defects', there are two main aspects to understand: their gravitational (or other particle) interactions and their dynamics. The gravity of domain walls is an interesting and rather more subtle topic than it might seem at first sight. Indeed, unlike all other defects (with the exception of global strings [10]), the wall's metric is not in general static [11,12], but admits a de Sitter-like expansion in its plane. Moreover, observers experience a repulsion from the wall, and there is a cosmological horizon at a finite proper distance from the defect's core. This horizon is a consequence of the choice of coordinates, and in a different set of coordinates [13] the wall has the appearance of a bubble which contracts in from infinite radius to some minimum radius, then re-expands, undergoing uniform acceleration from the origin. The 'horizon' is then simply the lightcone of the origin in these coordinates, and is somewhat similar to the horizon of Rindler spacetime. These results were originally obtained for infinitesimally thin walls, using the hypersurface formalism developed by Israel [14], but can be shown to be robust as an approximate description of a

thick domain wall by a perturbative expansion in the thickness of the wall [15, 16], or within the context of a fully nonlinear treatment of a scalar field coupled to gravity [17.

The crucial physical difference, then, of the self-gravitating domain wall spacetime, is the presence of the cosmological horizon, which introduces a second length scale into the system. Ordinarily, a defect possesses one length scale, its thickness $w$, however, the distance to the event horizon of the domain wall gives another length scale, $u_{\mathrm{h}}$, which can be compared to $w$. These lengths are given in terms of the coupling constants of the theory and, as taking a thin wall limit turns out to be a very artificial construction in terms of these underlying parameters, it becomes pertinent to examine both the gravity and dynamics for a thick 
domain wall.

The dynamics of topological defects are typically extremely nonlinear, and their study is usually carried out in the so-called Nambu approximation, where the full-field theory action of the model in which the defects arise is replaced by an approximation based on the degrees of freedom of the defect's core. At first sight, this looks like a perfectly reasonable approximation, since - even for finite-sized defects, such as string loops and wall bubblesthe thickness of the defect is typically many orders of magnitude smaller than its size. It must be noted however, that the Nambu action is only a leading order approximation of the real action, and is obtained in the limit $\alpha \rightarrow 0$, where $\alpha \propto K w$ is proportional to the defect's typical curvature and thickness [18 21]. However, it is often the case that the small-scale structure of defects (when $\alpha$ can be significant) is of particular importance when considering their impact. It seems that quite generically these are the points at which the defects lose most of their energy (by Higgs, gravitational or possibly other types of radiation, depending on the type of defect) and the properties of the small-scale therefore have a direct impact on the defects' lifespan, which in turn dictates the possible cosmological implications they can have.

The first attempts to derive effective actions for walls [19 21] naturally neglected gravity, in the sense that neither curvature of the background spacetime nor the self-gravity of the wall were considered. The effect of background spacetime curvature was considered (using the effective action method discussed below) in [22], and the self-gravity of the wall for a very special trajectory was considered in [20]; however, the motion of a fully self-gravitating thick domain wall (where the curvature of spacetime is that induced by the wall) has not to date been considered. In this paper, we address this problem, namely, using the $\lambda \Phi^{4}$ field theory, we examine the dynamics of a thick, self-gravitating kink domain wall solution.

There are essentially two methods which have been used to obtain the effective motion of a thick defect, both of which involve in some way an expansion of the fields around a well-known solution (such as the hyperbolic tangent kink for a $\lambda \Phi^{4}$ wall). The first approach consists of replacing the solution in the action and integrating out perpendicular to the defect, which yields an effective action based on the defect's core. This method has been employed for instance in [18,20 22]. The second approach consists of examining the field equations perturbatively in a relevant parameter (or parameters) such as $\alpha$, with the equations of motion to a particular order arising as an integrability condition (see for instance [20]). Here we use this latter method, adapted to take the wall's self-gravity into account. The reason for this choice is in fact related to the inclusion of gravity in the problem; the motion of the core of the defect interacts with massless degrees of freedom in the bulk (the graviton) and hence a correct application of the effective action method is less transparent.

The layout of the paper is as follows. In the next section we present the model, then briefly introduce the Gauss-Codazzi formalism and derive the Einstein equations in the corresponding " $3+1$ " notation. We end this section by reviewing our method in the case of a flat background spacetime. In section [II] we solve the field equations for the case $\alpha>\epsilon$ (where $\epsilon$ characterizes the gravitational interaction of the scalar field). In section IV] we discuss the particular case of a collapsing spherical wall, and we conclude in the last section. 


\section{THE GAUSS-CODAZZI FORMALISM}

Our starting point is the usual Goldstone matter Lagrangian,

$$
\mathcal{L}=\left(\nabla_{a} \Phi\right)^{2}-U(\Phi)
$$

where $\Phi$ is a real Higgs scalar field and $U(\Phi)$ is a symmetry-breaking potential which we take to be

$$
U(\Phi)=\lambda\left(\Phi^{2}-\eta^{2}\right)^{2}=\lambda \eta^{4} V\left(\frac{\Phi}{\eta}\right) .
$$

This model admits domain wall solutions, where the Higgs field tends to different vacua $\Phi= \pm \eta$ at, say, $u= \pm \infty$ for a flat wall. This implies the existence of a surface for which $\Phi=0$, and this surface defines the defect's core.

As in [17], we scale out the dimensionful parameters from the Lagrangian by defining

$$
X=\Phi / \eta \quad \epsilon=8 \pi G \eta^{2}
$$

$X$ now tends to \pm 1 at the vacua. The scalar and Einstein equations are

$$
\begin{aligned}
\square X+\frac{2}{w^{2}} X\left(X^{2}-1\right) & =0 \\
\mathcal{R}_{a b} & =\epsilon\left[2 X_{, a} X_{, b}-\frac{1}{w^{2}} g_{a b}\left(X^{2}-1\right)^{2}\right],
\end{aligned}
$$

where $\mathcal{R}_{a b}$ is the spacetime Ricci tensor, and $w$ is proportional to the inverse mass of the Higgs after the symmetry breaking, and therefore characterizes the width of topological defects within the theory. Note that according to (4b) $\epsilon$ characterizes the gravitational interaction of the Higgs field.

As mentioned in the introduction, our method for finding the effective dynamics of domain walls requires the expansion of the quantities appearing in the full equations of motion in powers of some small parameter. This can be achieved by splitting these quantities in their components parallel and perpendicular to the wall's worldvolume $\Sigma$, with the help of the Gauss-Codazzi formalism [20]. Before we start, a remark on our notation: although we shall generally use lowercase Latin indices $a, b, \ldots$, we may emphasize the parallel character of some index by using uppercase Latin letters $A, B, \ldots$ For instance, the coordinates parallel to the defect will be called $\sigma^{A}$. The proper distance from the wall will be denoted by $u$.

A domain wall's core (defined by the location of $X \equiv 0$ in the above model) is a threedimensional surface in four-dimensional spacetime, and consequently it admits a (spacelike) unit normal field denoted by $n^{a}$. This normal field can be regularly extended off the worldvolume by imposing $n^{a} \nabla_{a} n_{b}=0$, so that each surface of constant $u$ has a normal field $n^{a}$, a first fundamental tensor $h_{a b}$ and a second fundamental tensor $\hat{K}_{a b}$, the latter two being defined by

$$
h_{a b}=g_{a b}+n_{a} n_{b} \quad \hat{K}_{a b}=h_{a}^{c} \nabla_{c} n_{b} .
$$

$h_{a b}$ is the projection tensor onto the worldvolume (with our choice of coordinates, its parallel components are equal to the intrinsic metric's, and its perpendicular components vanish). 
$K_{a b}$ is the extrinsic curvature of $\Sigma$, which lies tangentially to the worldsheet and describes how the wall curves away from a hyperplane in spacetime. The metric of the wall spacetime is therefore written in this coordinate system as

$$
d s^{2}=h_{A B} d \sigma^{A} d \sigma^{B}-d u^{2} .
$$

The Gauss and Codazzi integrability conditions for the hypersurface generated by the wall's core are,

$$
\begin{gathered}
\hat{R}_{a b}=\mathcal{R}_{c d} h_{a}^{c} h_{b}^{d}+\hat{K}_{a c} \hat{K}_{b}^{c}-\hat{K} \hat{K}_{a b}+\mathcal{R}_{a c b d} n^{c} n^{d} \\
\hat{D}_{c} \hat{K}_{a}^{c}-\hat{D}_{a} \hat{K}=\hat{R}_{d e} n^{e} h_{a}^{d},
\end{gathered}
$$

where $\hat{D}_{a}=h_{a}^{c} \nabla_{c}$. Using these integrability conditions, the equations of motion (ब) can be rewritten

$$
\begin{aligned}
\frac{\partial h_{a b}}{\partial u} & =2 \hat{K}_{a b} \\
\frac{\partial \hat{K}_{a b}}{\partial u} & =-\epsilon\left(X^{2}-1\right)^{2} h_{a b}+\left[2 \hat{K}_{a c} \hat{K}_{b}^{c}-\hat{K} \hat{K}_{a b}-\hat{R}_{a b}\right]+2 \epsilon \hat{D}_{a} X \hat{D}_{b} X \\
\frac{\partial^{2} X}{\partial u^{2}}-2 X\left(X^{2}-1\right) & =-\hat{K} \frac{\partial X}{\partial u}+\hat{D}_{a} \hat{D}^{a} X \\
\frac{\partial \hat{K}}{\partial u} & =-\hat{K}_{a b}^{2}-\epsilon\left[2 X^{\prime 2}+\left(X^{2}-1\right)^{2}\right] \\
\hat{D}_{c} \hat{K}_{a}^{c}-\hat{D}_{a} \hat{K} & =2 \epsilon \frac{\partial X}{\partial u} \hat{D}_{a} X \\
\hat{R} & =\hat{K}_{a b}^{2}-\hat{K}^{2}+2 \epsilon\left[X^{\prime 2}-\left(X^{2}-1\right)^{2}+\hat{D}_{a} X \hat{D}^{a} X\right] .
\end{aligned}
$$

Here we have (without loss of generality) set $w=1$, which amounts to using wall rather than Planck units. The mean curvature $\hat{K}$ is the trace of $\hat{K}_{a b}$ and $\hat{R}_{a b}$ is the worldvolume Ricci tensor. Note that the equations for $\hat{K}_{a b}, \hat{K}$ and the derivatives of $\hat{K}$ in the wall correspond respectively to the " $A B$," "uu" and non-diagonal Einstein equations. The final equation results from the trace of (1) and turns out to be related to the integrability condition which gives the wall equation of motion.

We now need to identify the two key parameters in these equations, as well as the dependence of the variables on these parameters. Clearly the gravitational parameter appears explicitly in the equations, however, the parameter $\alpha$ characterising the motion of the wall is only implicit in the equations. The first step to identifying this parameter is to quantify what one means by 'motion' of the wall; this is encoded in the components of the extrinsic curvature on the wall core itself, since this tells us that the wall is curved in the ambient spacetime. We therefore set

$$
\alpha \sim\left|\hat{K}_{b}^{a}(u=0)\right| .
$$

Note that this is not a fundamental parameter of the theory, in that it is not given in terms of any coupling constants or masses, but simply represents the physical motion of the wall 
and enables the effect of that motion to be correctly considered. The extrinsic curvature $\hat{K}_{a b}$, therefore, has two main contributions: that of the motion of the wall core and that of the gravity of the wall. This can be estimated by considering the case of the plane-symmetric wall [17], for which $\alpha=0$, and

$$
\hat{K}_{a b}=A^{\prime} A \operatorname{diag}\left(1,-\mathrm{e}^{2 c t},-\mathrm{e}^{2 c t}\right),
$$

where $A(u)=1+\epsilon A_{1}(u)+O\left(\epsilon^{2}\right), c=2 \epsilon / 3+O\left(\epsilon^{2}\right)$. Clearly then, the components of $\hat{K}_{b}^{a}$ are $\mathrm{O}(\epsilon)$.

To summarise: in order to describe the motion of a domain wall in a curved spacetime we naturally have two parameters; one characterising the motion of the wall itself, $\alpha$, and one the curvature of the ambient spacetime, $\epsilon$. The basic procedure for determining the equation of motion of the wall is to solve (9) order by order in these parameters, investigating any constraints arising on the extrinsic curvature at each step. It is perhaps worthwhile briefly reviewing this process for $\epsilon=0$, since the methodology is very similar when gravity is included.

We begin by rescaling the extrinsic curvature and parallel coordinates via

$$
\begin{aligned}
\hat{K}_{a b} & =\alpha K_{a b} \\
\sigma^{A} & =x^{A} / \alpha .
\end{aligned}
$$

Since we are in flat space the Gauss identity (17) simplifies to,

$$
R_{a b}=K_{a c} K_{b}^{c}-K K_{a b}
$$

hence

$$
K_{a b}=\left.K_{a b}\right|_{0}+\left.\left.\alpha u K_{a}^{c}\right|_{0} K_{b c}\right|_{0}
$$

(where "|lo" indicates that a quantity is evaluated at the wall core) is actually an implicit exact solution to the $K$-equation. To order $\alpha$, we see that

$$
K=\left.K\right|_{0}-\left.\alpha u K_{a b}^{2}\right|_{0}
$$

and we can examine the $X$-equation (9c) by setting $X=X+\alpha X_{1}$, where $X_{0}=\tanh u$, finding

$$
\mathcal{D} X_{1} \equiv X_{1}^{\prime \prime}-2 X_{1}\left(3 X_{0}^{2}-1\right)=-\left.K\right|_{0} X_{0}^{\prime}
$$

Ordinarily, we might expect to be able to write the solution $X_{1}$ in terms of the basis of eigenfunctions of the operator $\mathcal{D}$, however, we cannot do this directly, since $X_{0}^{\prime}$ is in fact the zero mode of $\mathcal{D}$. We are therefore forced to either deduce that $\left.K\right|_{0}=0$, or, we can take the approach of reference 21] and remove the requirement that the equations of motion be regular at the wall. Since we are looking for freely moving wall trajectories, we will take the former approach, which can be summed up as an 'integrability requirement': multiplying both sides of (16) with $X_{0}^{\prime}$, and integrating over $\mathbb{R}$ implies

$$
\left(X_{1}^{\prime} X_{0}^{\prime}-X_{0}^{\prime \prime} X_{1}\right)_{-\infty}^{\infty}=\left.\int_{-\infty}^{\infty} K_{0}\right|_{0}\left(X_{0}^{\prime}\right)^{2} d u
$$


In order for $X_{1}$ to have the appropriate asymptotic behavior for large $u$ it follows that

$$
\left.K_{0}\right|_{0}=0 \quad\left(\Rightarrow X_{1}=0\right) .
$$

Equation (18) is of course the Nambu equation $\left.K\right|_{0}=0$, which simply means that the core of the defect, situated at $u=0$, follows the Nambu dynamics to zeroth order.

We can then repeat this process, expanding order by order to get

$$
\begin{aligned}
h_{a b} & =\left.h_{a b}\right|_{0}+\left.2 \alpha u K_{a b}\right|_{0}+\left.\left.\alpha^{2} u^{2} K_{a c}\right|_{0} K_{b}^{c}\right|_{0} \\
K & =\left.K\right|_{0}-\left.\alpha u K_{a b}^{2}\right|_{0}+\left.\left.\left.\alpha^{2} u^{2} K_{b}^{a}\right|_{0} K_{c}^{b}\right|_{0} K_{a}^{c}\right|_{0} \\
X & =X_{0}+\alpha X_{1}+\alpha^{2} X_{2},
\end{aligned}
$$

where $\left.K\right|_{0}=O\left(\alpha^{2}\right)$, and

$$
X_{2}=\operatorname{sech}^{2} u \int_{0}^{u} \cosh ^{4} u \int_{-\infty}^{u}\left(\left.u K_{a b}^{2}\right|_{0}\right) \operatorname{sech}^{4} u .
$$

This is sufficient to obtain the leading corrections to the Nambu action via the integrability constraints to third order. To third order (9d) gives

$$
\left[X_{3}^{\prime} X_{0}^{\prime}-X_{3} X_{0}^{\prime \prime}\right]^{\prime}=-K_{2} X_{0}^{\prime 2}=-\left(\left.K_{2}\right|_{0}+\left.\left.\left.u^{2} K_{b}^{a}\right|_{0} K_{c}^{b}\right|_{0} K_{a}^{c}\right|_{0}\right) X_{0}^{\prime 2},
$$

hence

$$
\left.K_{2}\right|_{0}=-\left.\left.\left.\frac{f_{2}(\infty)}{f_{0}(\infty)} K_{b}^{a}\right|_{0} K_{c}^{b}\right|_{0} K_{a}^{c}\right|_{0}=-\left.\left.\left.\left(\frac{\pi^{2}}{6}-1\right) K_{b}^{a}\right|_{0} K_{c}^{b}\right|_{0} K_{a}^{c}\right|_{0},
$$

where $f_{n}(u)=\int_{0}^{u} d u u^{n} \operatorname{sech}^{4} u$.

Note that this process of using the integrability condition to derive a constraint on the extrinsic curvature uses the $X$-equation to order $\mathrm{O}\left(\alpha^{n+1}\right)$ for a constraint on $K$ to order $\mathrm{O}\left(\alpha^{n}\right)$, and that this constraint only involves the even part of $K$, since any odd parts integrate to zero. Moreover, the $K$-equation (9d) shows that the even part of $K$ to order $\mathrm{O}\left(\alpha^{n}\right)$ depends on the odd part of $K_{a b}^{2}$ to order $\mathrm{O}\left(\alpha^{n-1}\right)$. Keeping this observation in mind prevents the unnecessary calculation of corrections to the geometric parameters.

\section{THE MOTION OF A WALL WITH GRAVITY}

In order to include gravity, we will make the initial assumption that gravity is subdominant to the motion of the wall, i.e. $\epsilon<\alpha$. Of course this need not always be the case, however, the derivation of the wall equations for $\epsilon>\alpha$ is almost identical to $\epsilon<\alpha$, and an expansion for general $\epsilon$ and $\alpha$ is so notationally cumbersome that we choose to present the analysis in this particular case for brevity and clarity.

First of all, note that the Ricci curvature of the wall is at least of order $\mathrm{O}\left(\alpha^{2}\right)$, as can be seen from (7), since the flat space Ricci curvature is given in terms of products of the extrinsic curvature, and the self-gravitating wall has $R_{a b}=O\left(\epsilon^{2}\right)$. We may therefore set

$$
\hat{R}_{a b}=\alpha^{2} R_{a b} .
$$


Rescaling the extrinsic curvature and parallel coordinates as in (12), and defining

$$
\delta=\frac{\epsilon}{\alpha},
$$

the equations of motion in the presence of gravity (here characterised by $\delta$ ) become

$$
\begin{aligned}
h_{a b}^{\prime} & =2 \alpha K_{a b} \\
K_{a b}^{\prime} & =-\delta\left(X^{2}-1\right)^{2} h_{a b}+\alpha\left(2 K_{a c} K_{b d} h^{c d}-R_{a b}-K K_{a b}\right)+2 \delta \alpha^{2} D_{a} X D_{b} X \\
X^{\prime \prime} & =2 X\left(X^{2}-1\right)-\alpha K X^{\prime}+\alpha^{2} D_{a} D^{a} X \\
K^{\prime} & =-\delta\left[2 X^{\prime 2}+\left(X^{2}-1\right)^{2}\right]-\alpha K_{a b}^{2} \\
D_{c} K^{c}{ }_{a}-D_{a} K & =2 \delta X^{\prime} D_{a} X \\
\alpha R & =\alpha K_{a b}^{2}-\alpha K^{2}+2 \delta\left[X^{\prime 2}-\left(X^{2}-1\right)^{2}+\alpha^{2} D_{a} X D^{a} X\right] .
\end{aligned}
$$

To solve these equations, we expand all quantities with respect to $\alpha$ :

$$
\begin{aligned}
X & =X_{0}+\alpha X_{1}+\alpha^{2} X_{2}+\cdots \\
h_{a b} & =h_{0 a b}+\alpha h_{1 a b}+\alpha^{2} h_{2 a b}+\cdots \\
K_{a b} & =K_{0 a b}+\alpha K_{1 a b}+\alpha^{2} K_{2 a b}+\cdots \\
R_{a b} & =R_{0 a b}+\alpha R_{1 a b}+\alpha^{2} R_{2 a b}+\cdots,
\end{aligned}
$$

which also implies similar series for the traces $K$ and $R$ with, for instance,

$$
K_{2}=K_{2 a b} h_{0}^{a b}-K_{1 a b} h_{1}^{a b}-K_{0 a b} h_{2}^{a b}+K_{0 a b} h_{1 c}^{a} h_{1}^{b c},
$$

where all indices are raised using $h_{0 a b}$. Note that we do not expand in a double series with $\delta$, since the presence of the $\alpha$ terms in the RHS of $(25 \mathrm{a}, \mathrm{c}, \mathrm{d})$ means that at any particular order in $\alpha$ the series expansion in $\delta$ terminates, as we can see from (28) below. We can now solve equations (25) order by order.

To zeroth order in $\alpha$ we obtain

$$
\begin{aligned}
h_{0 a b} & =\left.h_{0 a b}\right|_{0} \\
K_{0 a b} & =\left.K_{0 a b}\right|_{0}-\left.\delta f_{0}(u) h_{0 a b}\right|_{0} \\
K_{0} & =\left.K_{0}\right|_{0}-3 \delta f_{0}(u) \\
X_{0} & =\tanh u,
\end{aligned}
$$

and (251) is identically satisfied for $X_{0}=\tanh u$. We define

$$
\begin{aligned}
& f_{n}(u)=\int_{0}^{u} d u u^{n} V\left(X_{0}\right) \\
& F_{n}(u)=\int_{0}^{u} d u f_{n}(u) .
\end{aligned}
$$

Since $V(X)$ is an even function $f_{n}$ is odd (respectively, even) for $n$ even (respectively, odd). As a result $F_{n}$ is even (respectively, odd) for $n$ even (respectively, odd). By considering the derivative of (251) we can deduce that 


$$
R_{0}=\left.R_{0}\right|_{0} \quad \Rightarrow \quad R_{0 a b}=\left.R_{0 a b}\right|_{0} ;
$$

however, to find the actual value of $\left.R_{0}\right|_{0}$ we need to go to first order for $X$ (see equation (36) below).

To first order, we immediately obtain

$$
h_{1 a b}=\left.h_{1 a b}\right|_{0}+\left.2 u K_{0 a b}\right|_{0}-\left.2 \delta F_{0} h_{0 a b}\right|_{0}
$$

and

$$
X_{1}^{\prime \prime}-2 X_{1}\left(3 X_{0}^{2}-1\right)=-X_{0}^{\prime}\left[\left.K_{0}\right|_{0}-3 \delta f_{0}\right] .
$$

The integrability requirement then constrains $\left.K_{0}\right|_{0}=0$, and $X_{1}$ is found to be

$$
X_{1}=3 \delta X_{0}^{\prime} \int_{0}^{u} \frac{1}{X_{0}^{\prime 2}} \int_{-\infty}^{u} f_{0} X_{0}^{\prime}=-\frac{\delta}{6} \frac{3 u+\tanh u}{\cosh ^{2} u} .
$$

Note that $X_{1}$ is a correction due to the presence of gravity. Then

$$
\begin{aligned}
K_{1}=\left.K_{1}\right|_{0}-\left.u K_{0 a b}^{2}\right|_{0}-4 \delta X_{0}^{\prime} X_{1}-\delta^{2} G_{0} \\
K_{1 a b}=\left.K_{1 a b}\right|_{0}-\left.\delta f_{0} h_{1 a b}\right|_{0}+\left.\left.2 u K_{0 a c}\right|_{0} K_{0}{ }^{c}\right|_{0}-\left.\delta\left(2 f_{1}+F_{0}\right) K_{0 a b}\right|_{0} \\
\quad+\delta G_{1}(u) h_{0 a b}-\left.u R_{0 a b}\right|_{0}
\end{aligned}
$$

where the functions $G_{0}$ and $G_{1}$ are defined by

$$
\begin{aligned}
& G_{0}(u)=\int d u\left(4 \tanh u \operatorname{sech}^{2} u X_{1}+3 f_{0}^{2}\right) \\
& G_{1}(u)=2 f_{0} F_{0}+\int d u\left(4 \tanh u \operatorname{sech}^{2} u X_{1}-3 f_{0}^{2}\right),
\end{aligned}
$$

and are both of odd parity. Finally, from (25f) one obtains

$$
R_{0}=\left.K_{0 a b}^{2}\right|_{0}+2 \delta^{2}\left[2 X_{0}^{\prime} X_{1}^{\prime}-4 X_{0} X_{1}\left(X_{0}^{2}-1\right)-6 f_{0}^{2}\right]=\left.K_{0 a b}^{2}\right|_{0}-\frac{8}{3} \delta^{2} .
$$

At second order in $\alpha$, the equation for $X_{2}$ is

$$
\begin{aligned}
\mathcal{D} X_{2} & =6 X_{0} X_{1}^{2}-K_{1} X_{0}^{\prime}-K_{0} X_{1}^{\prime} \\
& =-\left.K_{1}\right|_{0} X_{0}^{\prime}+\left.u K_{0 a b}^{2}\right|_{0} X_{0}^{\prime}+6 X_{0} X_{1}^{2}+4 \delta X_{1} X_{0}^{\prime 2}+\delta^{2} G_{0} X_{0}^{\prime}+3 \delta X_{1}^{\prime} f_{0} .
\end{aligned}
$$

Since all but the first term on the RHS have odd parity, the integrability requirement once again constrains $\left.K_{1}\right|_{0}=0$, and we can solve for $X_{2}$ giving

$$
X_{2}=\operatorname{sech}^{2} u \int_{0}^{u} \cosh ^{4} u \int_{-\infty}^{u}\left(\left.u K_{a b}^{2}\right|_{0}\right) \operatorname{sech}^{4} u d u,
$$

which is an odd function with respect to $u$. Therefore, there are no corrections to the Nambu equation at first order just as in the case of flat spacetime. We do anticipate however that such corrections will appear at second order, and so proceed to calculate $K_{2}$. However, as we commented earlier, the constraints due to the integrability requirement only pertain to the even part of $K_{2}$, and this in turn depends on the odd part of $K_{2}^{\prime}$ : 


$$
\begin{aligned}
K_{2}^{\prime}= & -2 K_{0}^{a b} K_{1 a b}+2 K_{0 a b} K_{0 c}{ }^{b} h_{1}^{a c} \\
& -2 \delta\left[2 X_{0}^{\prime} X_{2}^{\prime}+X_{1}^{\prime 2}+2 X_{0} X_{2}\left(X_{0}^{2}-1\right)+X_{1}^{2}\left(3 X_{0}^{2}-1\right)\right] \\
= & \left.\left.2 u K_{0 a b}\right|_{0} R_{0}^{a b}\right|_{0}+[\text { even terms }] .
\end{aligned}
$$

Therefore,

$$
\left.K_{2}\right|_{\text {even }}=\left.K_{2}\right|_{0}+\left.\left.u^{2} K_{0 a b}\right|_{0} R_{0}^{a b}\right|_{0} .
$$

Now, examining the $X$-equation at order $\mathrm{O}\left(\alpha^{3}\right)$ we find

$$
\mathcal{D} X_{3}=2 X_{1}^{3}+12 X_{0} X_{1} X_{2}-K_{2} X_{0}^{\prime}-K_{1} X_{1}^{\prime}-K_{0} X_{2}^{\prime}
$$

and therefore the integrability requirement yields the constraint

$$
\left.K_{2}\right|_{0}=-\left.\left.\frac{f_{2}(\infty)}{f_{0}(\infty)} K_{0 a b}\right|_{0} R_{0}^{a b}\right|_{0}=-\left.\left.\left(\frac{\pi^{2}}{6}-1\right) K_{0 a b}\right|_{0} R_{0}^{a b}\right|_{0} .
$$

which gives us the first perturbation of the Nambu equation.

To summarize, the first corrections to the Nambu equations of motion (reversing the rescalings performed) appear at second order in $\alpha$ and are:

$$
\left.\hat{K}\right|_{0}=-\left.\left.\left(\frac{\pi^{2}}{6}-1\right) \hat{K}_{a b}\right|_{0} \hat{R}^{a b}\right|_{0}
$$

Although we obtained this result assuming $\epsilon<\alpha$, it is in fact quite general, since in a similar calculation for $\epsilon>\alpha$ rescaling with respect to $\epsilon$ gives exactly the same result. Clearly from (7) this correction has the correct flat space limit (22), and it would seem that the inclusion of gravity simply modifies the second order correction to the equations of motion, rather than causing a completely new correction to appear. Indeed the three dimensional Ricci curvature can be seen in (17) to relate to the extrinsic and background geometry of our spacetime.

A totally geodesic $\left(\left.K_{a b}\right|_{0}=0\right)$ solution such as the plane-symmetric wall always trivially satisfies (43). What is of real interest, however, is the existence of non-totally geodesic solutions verifying the perturbative equations of motion (43). A better understanding of the Nambu correction term will involve the computation of the three dimensional curvature tensor $\left.R_{0 a b}\right|_{0}$. This task is undertaken in the next section for the specific example of a collapsing spherical domain wall.

\section{THE COLLAPSE OF A SPHERICAL DOMAIN WALL}

In this section we apply the general equations of motion (43) to the case of a collapsing spherical domain wall. This is perhaps the simplest non-trivial example of a curved domain wall in curved space-time, i.e. where both of our perturbation parameters $\alpha$ and $\epsilon$ are not zero. The spherical domain wall has been already studied in different contexts using Israel's thin wall formalism [12,15,23]. A first attempt to study the thick case was undertaken in [20] but only using equations (22) valid for a flat space-time background. 
Consider, in a spherical system of coordinates $(t, r, \theta, \phi)$, a non-static scalar field representing a domain wall matter coupled to a spherically symmetric metric

$$
d s^{2}=A^{2}(t, r) d t^{2}-B^{2}(t, r) d r^{2}-r^{2} d \Omega_{I I}^{2} .
$$

The field equations one has to solve are the coupled Einstein and scalar equations, which can be written in a convenient way as

$$
\begin{aligned}
\frac{(A B)^{\prime}}{A B^{3}} & =\epsilon r\left(\frac{\dot{X}^{2}}{A^{2}}+\frac{X^{\prime 2}}{B^{2}}\right) \\
{\left[\left(1-\frac{1}{B^{2}}\right) r\right]^{\prime} } & =\epsilon r^{2}\left(\frac{\dot{X}^{2}}{A^{2}}+\frac{X^{\prime 2}}{B^{2}}+V(X)\right) \\
\frac{\dot{B}}{B} & =\epsilon r X^{\prime} \dot{X} \\
\frac{A^{\prime \prime}}{A B^{2}}-\frac{A^{\prime} B^{\prime}}{A B^{3}}-\frac{\ddot{B}}{B A^{2}}+\frac{\dot{B} \dot{A}}{B A^{3}}+\frac{2 A^{\prime}}{r A B^{2}} & =2 \epsilon \frac{\dot{X}^{2}}{A^{2}}-\epsilon V(X) \\
\square X+2 X\left(X^{2}-1\right) & =0 .
\end{aligned}
$$

For small values of $\epsilon \ll 1$, consider the field expansion,

$$
\begin{aligned}
X & =X_{0}+\epsilon X_{1}+O\left(\epsilon^{2}\right) \\
A & =1+\epsilon A_{1}+O\left(\epsilon^{2}\right) \\
B & =1+\epsilon B_{1}+O\left(\epsilon^{2}\right) .
\end{aligned}
$$

In order to solve (45) perturbatively one has to first solve the scalar equation (45e) to zeroth order in $\epsilon$. Then integrating out the Einstein equations (45a-45c) we can obtain the first order $\epsilon$ corrections for the metric $A_{1}$ and $B_{1}$. Let us first define the wall's position and discuss some general features about the solution before solving (45).

The location of the wall is defined by the zero of the Higgs field, and will have coordinates $X^{a}=(t, R(t), \theta, \phi)$. We start by making the observation that in order for the wall to be identifiably a topological kink solution, $R(t) \gg 1$, and without loss of generality, we can assume that $X<0$ (respectively, $X>0$ ) for $r<R(t)(r>R(t))$. (Note that these are not the Gauss-Codazzi gauge coordinates centered on the wall's core.)

We consider the following initial conditions which are compatible with the fact that, due to its spherical symmetry, the wall is not radiating:

$$
R(t=0)=R_{\mathrm{i}}, \quad \dot{R}(t=0)=0 .
$$

The wall's initial bending parameter is defined as $\alpha=1 / R_{\mathrm{i}}$.

For an inertial observer situated outside the wall, $r>R(t)$, so by Birkhoff's theorem the exterior metric is Schwarzschild:

$$
d s^{2}=\left(1-\frac{2 G M}{r}\right) d t^{2}-\left(1-\frac{2 G M}{r}\right)^{-1} d r^{2}+r^{2} d \Omega_{I I}^{2}, \quad r>R(t) .
$$

In the same way, inside the wall the spacetime metric is flat to a very good approximation. The wall's mass, as measured by a distant observer, is $M=4 \pi \sigma R_{\mathrm{i}}^{2}$ and the Schwarzchild radius is given by $r_{\mathrm{S}}=2 G M$. In order for $R_{\mathrm{i}}>r_{\mathrm{S}}$, one must impose the relation $\alpha>\epsilon$. 
Keeping these considerations in mind, let us now proceed with solving our field equations order by order in $\epsilon$ in the region close to the wall's core, $r \approx R(t)$. In order to do so, we must also expand the function $R(t)$, determining the wall's position, in powers of $\epsilon$,

$$
R(t)=R_{0}(t)+\epsilon R_{1}(t)+O\left(\epsilon^{2}\right) .
$$

Of course $R_{0}(t)$ will in fact be given in terms of a power series in $\alpha: R_{0}(t)=\mathcal{R}_{0}(t)+\alpha^{2} \mathcal{R}_{2}(t)$ etc.

To zeroth order in $\epsilon$, i.e. in a flat background spacetime, we can define the unit, exterior pointing, normal vector to the wall's core at $r=R_{0}(t)$ as

$$
n^{a}=\left(\dot{R}_{0}, 1,0,0\right) / \sqrt{1-\dot{R}_{0}^{2}}
$$

The intrinsic metric and extrinsic curvature components of the wall (at $r=R_{0}(t)$ ) are easily calculated in turn using (5). The extrinsic curvature for $\epsilon=0$ is then given by

$$
K_{b}^{a}=\frac{\ddot{R}_{0} \delta_{t}^{a} \delta_{b}^{t}}{\left(1-\dot{R}_{0}{ }^{2}\right)^{3 / 2}}+\frac{\delta_{\theta}^{a} \delta_{b}^{\theta}+\delta_{\phi}^{a} \delta_{b}^{\phi}}{R_{0} \sqrt{1-\dot{R}_{0}{ }^{2}}}
$$

and the equation of motion (using (22)) is

$$
\ddot{R}_{0}=-\frac{2}{R_{0}}\left(1-\dot{R}_{0}{ }^{2}\right)-\left(\frac{\pi^{2}}{6}-1\right)\left[\frac{\ddot{R}_{0}^{3}}{\left(1-\dot{R}_{0}^{2}\right)^{3}}+\frac{2}{R_{0}^{3}}\right]+O\left(\alpha^{3}\right) .
$$

This can be solved iteratively, giving to leading order

$$
\left(\frac{\mathcal{R}_{0}}{R_{\mathrm{i}}}\right)^{4}=1-\dot{\mathcal{R}}_{0}^{2}
$$

which can be solved analytically and numerically, as can the $\mathrm{O}\left(\alpha^{2}\right)$ correction (see [20] and [24,25]), giving the wall trajectory as shown in figure [1. To $\mathrm{O}\left(\alpha^{2}\right)$ we note that $R_{0}$ satisfies

$$
1-\dot{R}_{0}^{2}=\frac{R_{0}^{4}}{R_{\mathrm{i}}^{4}}\left[1-\frac{2 C}{R_{\mathrm{i}}^{2}}\left(1-\frac{R_{\mathrm{i}}^{6}}{R_{0}^{6}}\right)\right],
$$

where $C \equiv f_{2}(\infty) / f_{0}(\infty)=\pi^{2} / 6-1$. From (52) and (53) we note that $\frac{d^{n}}{d t^{n}} R_{0}=\mathrm{O}\left(\alpha^{n-1}\right)$.

In order to solve for the spacetime metric, we need $X_{0}$. From the work of the previous section we know that $X_{0}=\tanh u+\chi_{2}$, where $\chi_{2}$ is given from equation (20) as

$$
\chi_{2}=\frac{6}{R_{0}^{2}\left(1-\dot{R}_{0}^{2}\right)} \operatorname{sech}^{2} u \int_{0}^{u} \cosh ^{4} u \int_{-\infty}^{u} u \operatorname{sech}^{4} u,
$$

and is clearly of order $\mathrm{O}\left(\alpha^{2}\right)$. Here, $u$ is the proper distance from the wall. Using (50) we note that

$$
\begin{aligned}
& t=t^{\prime}+\frac{u \dot{R}_{0}\left(t^{\prime}\right)}{\sqrt{1-{\dot{R_{0}}}^{2}\left(t^{\prime}\right)}} \\
& r=R_{0}\left(t^{\prime}\right)+\frac{u}{\sqrt{1-\dot{R}_{0}{ }^{2}\left(t^{\prime}\right)}},
\end{aligned}
$$




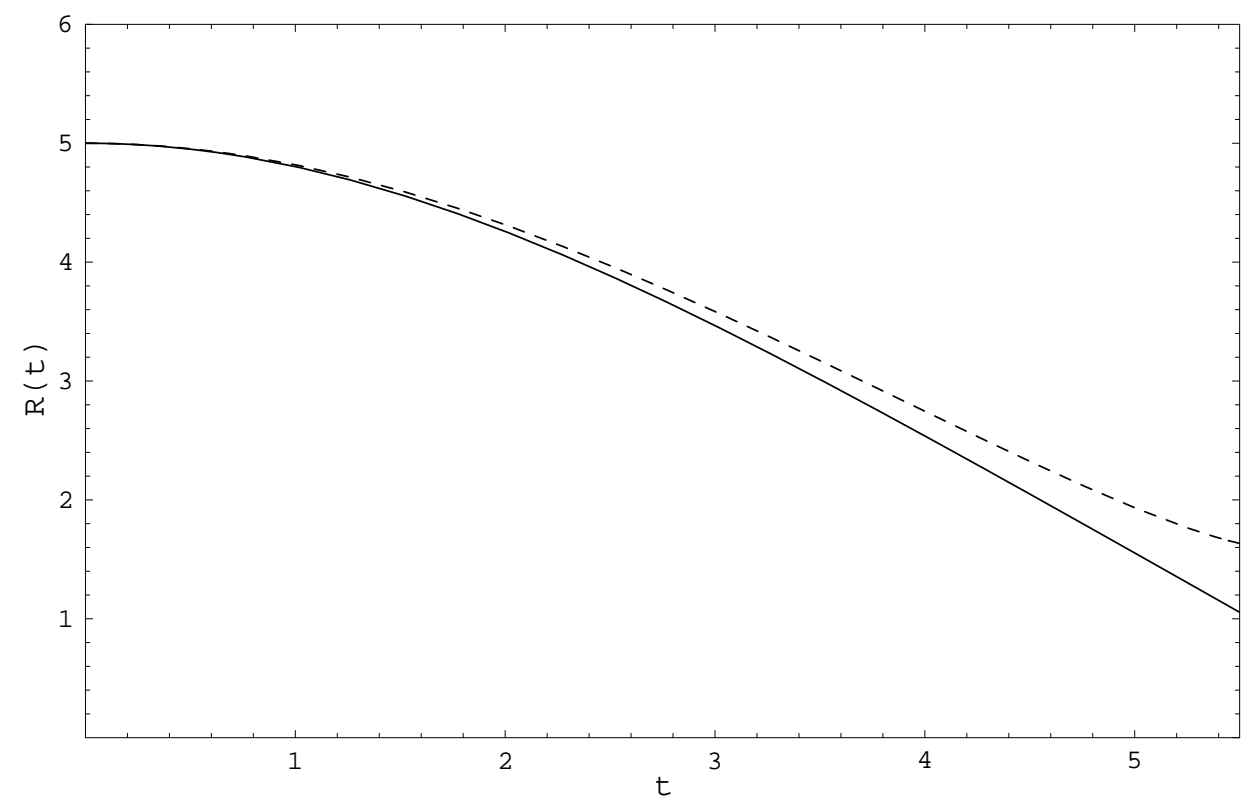

FIG. 1. The trajectory for the collapsing wall in flat spacetime. The solid line is the leading order Nambu trajectory, and the dashed line the trajectory including $\mathrm{O}\left(\alpha^{2}\right)$ corrections. For illustrative purposes the rather large value of $\alpha=1 / 5$ has been chosen.

where $\left(t^{\prime}, u\right)$ are the coordinates of the point $(t, r)$ in Gaussian Normal gauge.

In order to find the metric, first note that we can directly integrate (45a) and (45b) implicitly, finding

$$
\begin{aligned}
& B_{1}=\frac{1}{2 r} \int_{0}^{r} r^{2}\left[\left(\frac{1+\dot{R}_{0}^{2}\left(t^{\prime}\right)}{1-\dot{R}_{0}^{2}\left(t^{\prime}\right)}\right) X_{0}^{\prime 2}+V\left(X_{0}\right)\right] d r \\
& A_{1}=-\frac{1}{2 r} \int_{0}^{r} r^{2}\left[\left(\frac{1+\dot{R}_{0}^{2}\left(t^{\prime}\right)}{1-\dot{R}_{0}^{2}\left(t^{\prime}\right)}\right) X_{0}^{\prime 2}+V\left(X_{0}\right)\right] d r+\int_{0}^{r} r\left(\frac{1+\dot{R}_{0}^{2}\left(t^{\prime}\right)}{1-\dot{R}_{0}^{2}\left(t^{\prime}\right)}\right) X_{0}^{\prime 2} d r
\end{aligned}
$$

where we have used (56) to obtain $\dot{u}^{2}+u^{\prime 2}=\left[1+\dot{R}_{0}^{2}\left(t^{\prime}\right)\right] /\left[1-\dot{R}_{0}^{2}\left(t^{\prime}\right)\right]$. We may now substitute the form of $X_{0}\left(u, t^{\prime}\right)$ to the required order, replace the $r$-integral by a $u$-integral along a line $t=$ const, then Taylor expand $t^{\prime}$ around $u=0$ to the required order in $\alpha$. For example, $B_{1}$ gives the mass function via $B^{-2}=1-2 G M(r) / r \Rightarrow G M(r)=\epsilon r B_{1}$. Computing $B_{1}$ from the above expression to $\mathrm{O}\left(\alpha^{2}\right)$ yields

$$
G M(r)=\frac{\epsilon R_{0}^{2}}{\sqrt{1-\dot{R}_{0}^{2}}} \tilde{f}_{0}(u)+2 \epsilon \frac{R_{\mathrm{i}}^{4}}{R_{0}^{3}} \tilde{f}_{1}(u)+\epsilon R_{\mathrm{i}}^{2} \operatorname{sech}^{2} u \chi_{2}(u)+\epsilon \frac{R_{\mathrm{i}}^{6}}{R_{0}^{6}}\left[\tilde{f}_{2}(u)+6 \dot{R}_{0}^{2} u \tilde{f}_{1}(u)\right]
$$

where we have put

$$
\tilde{f}_{n}(u)=\int_{-\infty}^{u} d u u^{n} V\left(X_{0}\right)
$$

This gives the ADM mass (using 54) as 


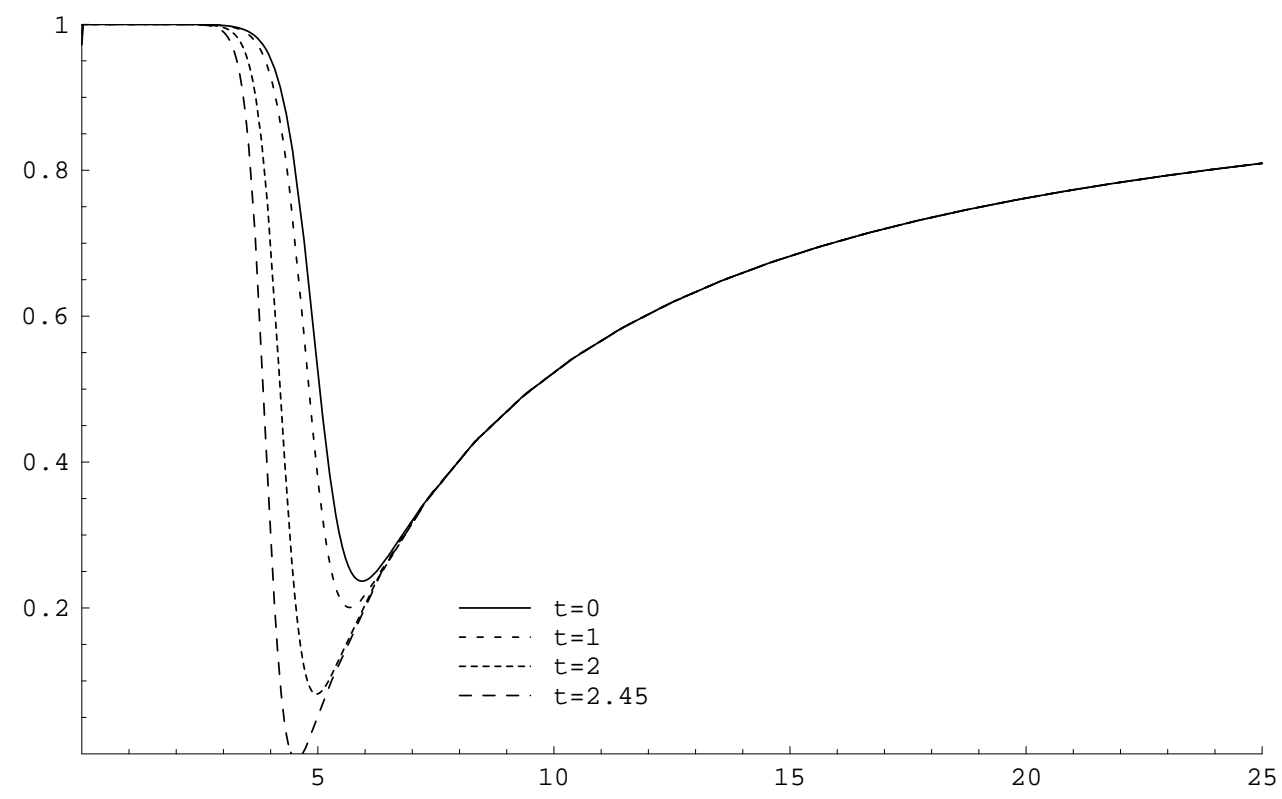

FIG. 2. The $r-r$ component of the metric for the collapsing wall solution of figure 1 for various values of $t$ up to $t_{c}$, the critical value of $t$ for which the wall reaches its Schwarzschild radius. Again, for illustrative purposes, the rather large value of $\epsilon=1 / 14$ has been chosen.

$$
G M_{\mathrm{ADM}}=\lim _{r \rightarrow \infty} \epsilon r B_{1}=\frac{\epsilon R_{0}^{2}}{\sqrt{1-\dot{R}_{0}^{2}}} \tilde{f}_{0}(\infty)+\epsilon \frac{R_{\mathrm{i}}^{6}}{R_{0}^{6}} \tilde{f}_{2}(\infty)=\frac{4}{3} \epsilon\left(R_{\mathrm{i}}^{2}+\frac{\pi^{2}}{6}-1\right)
$$

which is indeed constant, and agrees to leading order with the expected result. Figure 2 shows the evolution of the $g_{r r}$ metric component up to the formation of a black hole.

The gravitational Nambu equation for $\epsilon \neq 0$ at $u=0$ is given by

$$
\ddot{R}+\frac{2}{R} n A^{2}+\left(A^{\prime} A+\dot{R} \dot{B} B\right) n+\dot{R}^{2}\left(\frac{B^{\prime}}{B}-\frac{A^{\prime}}{A}\right)+\dot{R}\left(\frac{\dot{B}}{B}-\frac{\dot{A}}{A}\right)=O\left(\alpha^{2} \epsilon\right),
$$

where $n=B^{-2}-\dot{R}^{2} A^{-2}$. Note that the gravitational correction to $A$ and $B$ is $\mathrm{O}(\delta)$ not $\mathrm{O}(\epsilon)$, where $\delta=\epsilon / \alpha$ was defined in (24). This means that gravitational corrections to the wall motion potentially could appear at $\mathrm{O}(\delta)$. Since the flat space wall equations are intially $\mathrm{O}(\alpha)$, we could have the catastrophic situation that adding gravity swamps the wall motion, and has a superdominant effect. In fact, this turns out not to be the case. The computation of the $A$ and $B$ contributions to $\mathrm{O}(\delta)$ in (61) shows that they cancel. Instead, the leading order correction appears at $\mathrm{O}(\delta \alpha=\epsilon)$, which is therefore subdominant to the flat space motion, and is plotted in figure 3

$$
\ddot{R}=-\frac{2}{R}\left(1-\dot{R}^{2}\right)+2 \epsilon\left(1-\dot{R}^{2}\right)^{3 / 2}+\frac{2 \epsilon(4 \ln 2-1) \dot{R}^{2}}{3 R}\left(1-6 \dot{R}^{2}+3 \dot{R}^{4}\right)
$$

Note that we have included the $\mathrm{O}(\alpha \epsilon)$ correction, since it is the same order as the finite width correction, however, this has not been used in the computation of the corrected 
Nambu trajectory in figure 3. We should stress that this correction does not mean that the wall trajectory is no longer Nambu, it simply gives the right gravitational alteration to the trajectory to allow the wall to remain a minimal surface in the now curved spacetime. What is important however, is that the correction is subdominant to the flat space motion and is a simple 'nudge'; there is no evidence that adding gravity gives any catastrophic effect which could force a trajectory to be totally geodesic.

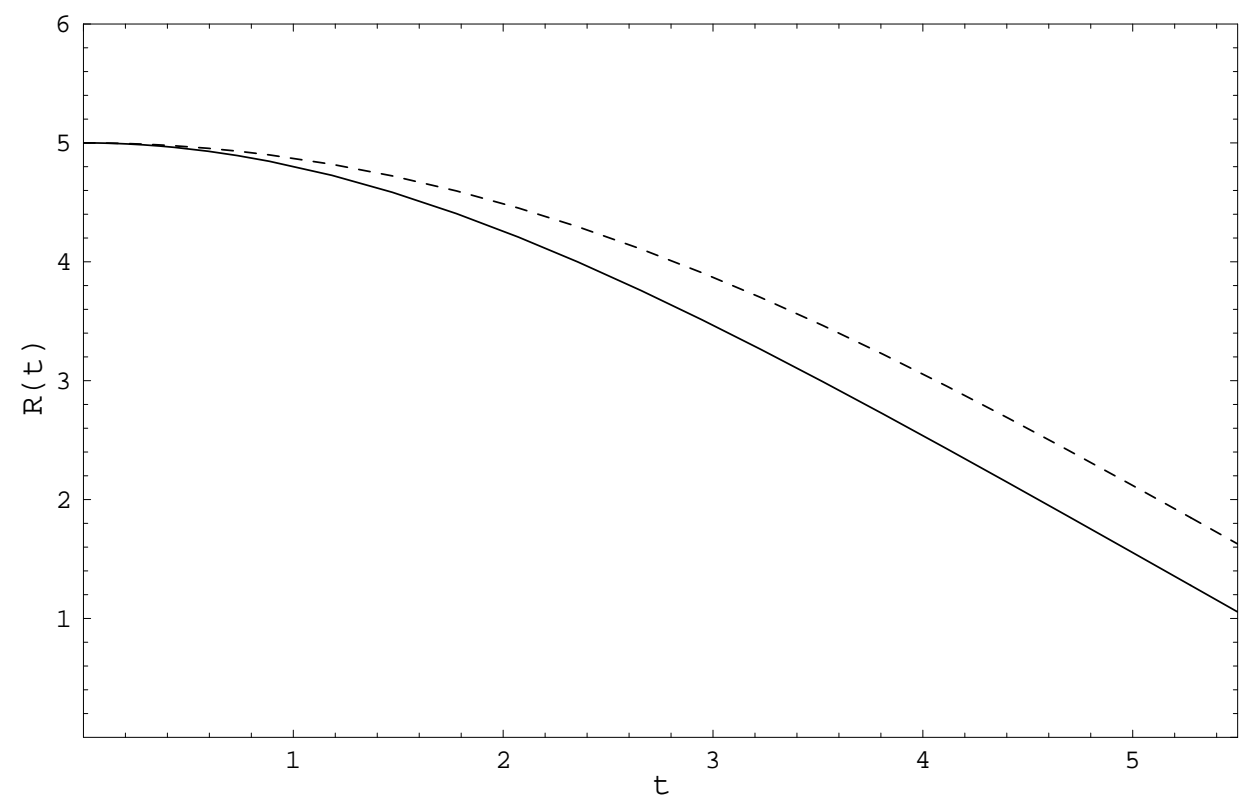

FIG. 3. A comparison of the leading order (i.e. Nambu) flat space bubble trajectory (solid line) and the leading order (Nambu) trajectory in the presence of gravity. As before, for illustrative purposes the rather large values of $\alpha=1 / 5$ and $\epsilon=1 / 14$ have been chosen. Although the bubble actually reaches its Schwarzschild radius at $t \simeq 2.45$ (see fig 21), we have chosen the $t$-range for direct comparison with figure 1 .

What is of real interest to us however, at least in the context of our general equation (43), is the correction to the Nambu motion. Indeed, having a particular example, we can explicitly calculate $R_{a b}$ from Gauss's equation (7). Since we have not rescaled our quantities here, we can check that the correction term is indeed of the right order, and get an idea of the physical implication that the correction induces (at least in this particular example). We obtain,

$$
\hat{K}_{0}=\frac{\pi^{2}-6}{\left(1-\dot{R}_{0}{ }^{2}\right)^{3 / 2}}\left(\frac{1}{R_{0}^{3}}+\frac{\epsilon}{R_{0}} \frac{\dot{R}_{0}{ }^{2}\left(1+\dot{R}_{0}{ }^{2}\right)}{\left(1-\dot{R}_{0}{ }^{2}\right)^{2}}\right)
$$

The first term is a correction term of order $\mathrm{O}\left(\alpha^{3}\right)$ due to the bending of the wall, already present in flat space-time as predicted in [20]. The second correction term is due to selfgravity appearing at $\mathrm{O}(\alpha \epsilon)$, as we were indeed expecting (for a spherical wall $\alpha>\epsilon$ ). Note that the finite width gravitational correction can dominate the extrinsic curvature correction, 
depending on the relative magnitudes of $\epsilon$ and $\alpha^{2}$. Finally from the overall positive sign we can deduce that the corrections to the Nambu motion induce a slowdown of the wall's collapse.

Using (62) and (63) we can write the full equation of motion (i.e. including the dominant deviation to Nambu motion) for a collapsing spherical wall up to and including order $\alpha \epsilon$,

$$
\begin{aligned}
\ddot{R}= & -\frac{2}{R}\left(1-\dot{R}^{2}\right)+2 \epsilon\left(1-\dot{R}^{2}\right)^{3 / 2}+\frac{2 \epsilon(4 \ln 2-1) \dot{R}^{2}}{3 R}\left(1-6 \dot{R}^{2}+3 \dot{R}^{4}\right) \\
& +\left(\pi^{2}-6\right) \frac{\epsilon}{R} \frac{\dot{R}^{2}\left(1+\dot{R}^{2}\right)}{\left(1-\dot{R}^{2}\right)^{2}} .
\end{aligned}
$$

Before leaving the collapsing wall, it is worthwhile comparing our thick wall calculation with the results of Ipser and Sikivie, [12], obtained for the collapsing thin wall. In order to obtain the Israel thin wall approximation one should reintroduce the width parameter $w$ which has been set equal to unity throughout this analysis. Then $\alpha=w / R$ (where $\mathrm{R}$ is a typical radius of curvature of the wall) is a dimensionless parameter. The Israel limit is then obtained by letting $\alpha$ and $\epsilon$ tend to zero, while keeping their quotient $\delta$ fixed. This amounts to keeping the horizon distance from the wall finite i.e., keeping the wall self-gravitating (as one should in this formalism). This also gives us the correct limit without involving the normal coordinate $u$ in the limiting procedure. Taking this limit in (64) gives

$$
\ddot{R}=-\frac{2}{R}\left(1-\dot{R}^{2}\right)+2 \delta\left(1-\dot{R}^{2}\right)^{3 / 2}
$$

which is obviously only correct to $\mathrm{O}(\delta)$. The translation to the calculation of Ipser and Sikivie is not direct, since our coordinates correspond to the interior coordinates of their bubble, however, once the correct correspondence is made, we do indeed find precise agreement to order $\mathrm{O}(\delta)=\mathrm{O}(G \sigma)$.

\section{CONCLUSIONS}

We have obtained a general equation of motion (43) describing a moving wall in curved spacetime. This was achieved by analytically solving the Einstein and scalar matter field equations order by order with respect to two parameters: the wall's bending parameter, $\alpha$, and the gravitational strength parameter, $\epsilon$, expressing the curving of spacetime. We then considered a particular example, the collapsing spherical domain wall, which is perhaps the simplest non-trivial example with both parameters different from zero. In the context of this example we found that the corrections to the flat space Nambu motion tended to slow down the wall's collapse.

Throughout this paper we have considered a thick wall with scalar (Higgs) matter. This was done in order to examine the problem in its analytic (with respect to the spacetime metric) and most general context and also in order to pick up finite width gravitational

corrections. As we discussed at the end of the previous section, in order to obtain the Israel thin wall approximation one takes the limit $\alpha \rightarrow 0$ and $\epsilon \rightarrow 0$ keeping $\delta$ fixed. Although the spherical wall was only explored to $\mathrm{O}(\delta)$, the arguments in section [II show in all generality that the corrections to the Nambu equation of motion $K=0$ are finite width corrections, 
and hence disappear in the thin wall limit, giving simply gravitational corrections to the flat space trajectory.

A totally geodesic $\left(\left.K_{a b}\right|_{0}=0\right)$ solution such as the plane-symmetric wall always trivially satisfies (43). With respect to the physical motion of the wall a totally geodesic solution is trivial and in particular a totally geodesic wall will not emit gravitational waves. What is of real interest physically, is the existence of non-totally geodesic solutions such as the spherical domain wall satisfying (43). There have been claims however (see for the related example of a cosmic string, [26], and references therein), that the presence of gravity in general constrains a defect's core to be totally geodesic. For a domain wall in particular it has been claimed that the presence of gravity induces the wall to lose its dynamical degree of freedom and not to radiate [27]. We should stress that throughout our treatment this constraint has not appeared and gravity affects the motion of the defect in a very natural way in the sense that corrections to the Nambu motion appear at the same order as in the case of a flat background spacetime. We suspect that the reason for this discrepancy is that in [27] rather specific asymptotic boundary conditions have been placed on the spacetime, namely that it asymptote the static planar domain wall solution. If, however, a wall is oscillating, we expect that its effective equation of state will change, analogous to that of the wiggly cosmic string [28], which will change the spacetime metric even asymptotically. Mathematically, this can be seen via the divergent behaviour of the metric perturbations in the Gaussian Normal gauge due to proper motion of the wall, and was discussed in the context of higher dimensional domain walls in 29]. Following [28], we expect that the equation of state of the wall will have the form $\sigma T^{2}=\sigma_{0}^{3}$, or, that perturbatively the effect on the energymomentum tensor of the wall will be to increase the energy by $\delta \sigma$, and decrease the tension by $\delta \sigma / 2$. Note that this perturbation is tracefree, and localised on the wall. In fact if we regard our domain wall as a $2+1$-dimensional universe, this energy momentum would be that appropriate to a radiation dominated cosmology. Such systems have been explored in a different context in, for example, [30].

As a final point it should be clear that although we have considered a four-dimensional spacetime our equations of motion are valid for any $(n-2)$-brane of an $n$-dimensional spacetime. Our analysis however relies heavily on the fact that the wall is a hypersurface of the ambient spacetime i.e. that codimension is one. If we were to consider dynamics of strings for instance, the picture could in principle be quite different.

\section{ACKNOWLEDGEMENTS}

We would like to thank Roberto Emparan, David Langlois, Joao Magueijo and Valery Rubakov for useful discussions. F.B. is supported by a FAPESP grant; C.C. is supported by PPARC and R.G. is supported by the Royal Society. 


\section{REFERENCES}

[1] A.Vilenkin and E.P.S.Shellard, Cosmic strings and other topological defects (Cambridge: Cambridge University Press 1994).

[2] A.Albrecht, R.A.Battye and J.Robinson, Phys. Rev. Lett. 794736 (1997). astroph/9707129].

U.L.Pen, U.Seljak and N.Turok, Phys. Rev. Lett. 791611 (1997). astro-ph/9704165.

[3] P.Ferreira, J.Magueijo and K.M.Gorski, Astrophys. J. 503 L1 (1998). astroph/9803256].

J.Pando, D.Valls-Gabaud and L.-Z.Fang, Phys. Rev. Lett. 814568 (1998). astroph/9810165.

[4] A.J.Banday, S.Zaroubi and K.M.Gorski, On the non-gaussianity observed in the COBEDMR sky maps, astro-ph/9908070.

[5] R.A.Battye, J.Robinson and A.Albrecht. Phys. Rev. Lett. 804847 (1998). astroph/9711336].

[6] The Supernova Cosmology Project (S.Perlmutter et.al.), Measurements of $\Omega$ and $\Lambda$ from 42 high redshift supernovae, astro-ph/9812133. http: //www-supernova. lbl.gov/

[7] Ya.B.Zel'dovich, I.Yu.Kobzarev and L.N.Okun, Sov. Phys.-JETP 401 (1974).

[8] V.A.Rubakov and M.E.Shaposhnikov, Phys. Lett. 125B 136 (1983).

[9] L.Randall and R.Sundrum, Phys. Rev. Lett. 833370 (1999). hep-ph/9905221 Phys. Rev. Lett. 834690 (1999). hep-th/9906064

[10] R.Gregory, Phys. Rev. D54 4995 (1996). gr-qc/9606002. Nonsingular global string compactifications, hep-th/9911015.

[11] A.Vilenkin, Phys. Lett. 133B 177 (1983).

[12] J.Ipser and P.Sikivie, Phys. Rev. D30 712 (1984).

[13] G.W.Gibbons, Nucl. Phys. B394 3 (1993).

[14] W.Israel, Nuovo Cim. 44B 1 (1966). Erratum: Nuovo Cim. 48B 463 (1967).

[15] L.M.Widrow, Phys. Rev. D39 3571 (1989). Phys. Rev. D39 3576 (1989).

[16] D.Garfinkle and R.Gregory, Phys. Rev. D41 1889 (1990).

[17] F.Bonjour, C.Charmousis and R.Gregory, Class. Quant. Grav. 162427 (1999). grqc/9902081.

[18] K.-I.Maeda and N.Turok, Phys. Lett. 202B 376 (1988).

R.Gregory, Phys. Lett. 206B 199 (1988). Phys. Rev. D43 520 (1991).

[19] V.Silveira, Phys. Rev. D38 3823 (1988).

[20] R.Gregory, D.Haws and D.Garfinkle, Phys. Rev. D42 343 (1990).

[21] B.Carter and R.Gregory, Phys. Rev. D51 5839 (1995). hep-th/9410095.

[22] C.Barrabès, B.Boisseau and M.Sakellariadou, Phys. Rev. D49 2734 (1994). grqc/9307008.

[23] K.Maeda, Gen. Rel. Grav. 18931 (1986).

[24] P.S.Letelier, Phys. Rev. D41 1333 (1990).

H.Arodz and A.L.Larsen, Phys. Rev. D49 4154 (1994).

[25] V.A.Berezin, V.A.Kuzmin and I.I.Tkachev, Phys. Lett. 120B 91 (1993). Phys. Rev. D36 2919 (1987).

[26] B.Boisseau, C.Charmousis and B.Linet Phys. Rev. D55 616 (1997). gr-qc/9607029

[27] H.Kodama, H.Ishihara and Y.Fujiwara Phys. Rev. D50 7292 (1994). gr-qc/9401007 
A.Ishibashi and H.Ishihara Phys. Rev. D56 3446 (1997). gr-qc/9704058]

A.Ishibashi and H.Ishihara Phys. Rev. D60 124016 (1999). gr-qc/9802036

[28] A.Vilenkin, Phys. Rev. D41 3038 (1990).

B.Carter Phys. Rev. D41 3869 (1990).

[29] C.Charmousis, R.Gregory and V.A.Rubakov, Wave function of the radion in a brane world, hep-th/9912160.

[30] P.Binetruy, C.Deffayet and D.Langlois, Nonconventional cosmology from a brane universe, hep-th/9905012.

C.Csaki, M.Graesser, C.Kolda and J.Terning, Phys. Lett. 462B 34 (1999). hepph/9906513 